\title{
Giant Ulcerative Hemangioma in a Newborn
}

\author{
Chokoeva $\mathrm{AA}^{1}$, Enache $\mathbf{O A}^{2}$, Wollina $\mathbf{U}^{3}$, Tchernev $\mathrm{G}^{4 *}$ \\ ${ }^{1}$ Onkoderma-Policlinic for Dermatology and Dermatologic Surgery, General Skobelev ,Sofia, Bulgaria \\ ${ }^{2}$ Emergency Clinical County Hospital Craiova, Craiova, Romania \\ ${ }^{3}$ Department of Dermatology and Allergology, Academic Teaching Hospital Dresden-Friedrichstadt, Dresden, Germany \\ ${ }^{4}$ Policlinic for Dermatology and Venereology, University Hospital Lozenetz, Sofia, Bulgaria
}

Received: November 04, 2015; Accepted: November 10, 2015; Published: November 17, 2015

*Corresponding author: Assoc. Prof. Georgi Tchernev, Policlinic for Dermatology and Venereology, University Hospital Lozenetz, Koziak street 1, 1407 Sofia, Bulgaria, Tel: +359 885588 424; E-mail:georgi_tchernev@yahoo.de

\begin{abstract}
Infantile hemangiomas $(\mathrm{IH})$ are the most common benign soft tissue vascular tumors in early childhood with an incidence of up to $10 \%-12 \%$, which affected most commonly the face and neck area, followed by the trunk and extremities, which ususally appears within the first weeks of life. IF represent true neoplasms of endothelial cells which should be sharply differentiated from vascular malformations, caused by dysfunction in embryogenesis and vasculogenesis. Although that the prevalence of IH tend to regress spontaneously, between 10 and $15 \%$ are at risk for complications, especially during the proliferative phase of the disease, which could cause impairment of vital functions or permanent cosmetic disfigurement. The major therapeutic dilemma consist in physician's decision to treat the disease in the first weeks of life, considering the high rate of advanced effects associated with the approved treatment modalities or to wait the possible spontaneous regression, considering the potential risk of complications. Current study obtained a significant difference between the expression of $\mathrm{p} 16$ protein in the proliferating hemangiomas and in the involuting hemangiomas, suggesting it as a potential prognostic marker which could determine the further therapeutic behavior. We present a rare case of extra facial giant infantile hemangioma in a newborn, which still remains a therapeutic challenge.
\end{abstract}

Keywords: Hemangioma; Ulceration; Conservative approach; Beta blockers

\section{Introduction}

Infantile hemangiomas (IH) are the most common benign soft tissue vascular tumors in early childhood with an incidence of $10 \%$ to $12 \%$ [1]. IH are seen more frequently among white neonates, twins, girls, premature infants, as higher maternal age is also considered as a risk factor for higher incidence $[1,2]$. The head-and-neck region is most often affected (60\%), followed by the trunk (25\%) and the extremities (15\%) [2].

IH's usually occurs within the first weeks of life, which required a differentiation between Congenital Hemangiomas which are fully formed at birth [3]. Their histological and immunohistochemical findings separate them as a clinical entity with different therapeutic modalities [3].

The first classification of congenital vascular tumors and malformations was described in 1982 by Mulliken and Glowacki, but nowadays it seems unsatisfactory in regard to the interchangeable use of the terms hemangioma and vascular malformation $[2,4]$. The Greek suffix "oma" highlighted the cellular proliferation component of a tumor, which is not suitable for malformations [2]. From a histopathological point of view, hemangiomas represent true neoplasms of endothelial cells which should be sharply differentiated from vascular malformations, being a localized defect of vascular morphogenesis, caused by dysfunction in embryogenesis and vasculogenesis [2]. Current classification differentiating vascular tumors from vascular malformations based on their clinical appearance, radiological features, pathological features and biological behavior [2].

Although the etiology and pathogenesis of hemangiomas are not fully understood, many theories have been postulated to explain their origin including suggestions of placental origin, somatic mutation of vascular endothelial growth factor receptors, intrinsic defects and extrinsic factors (tissue hypoxia and developmental field disturbances), as well as an imbalance between pro- and antiangiogenic factors could be observed in infantile hemangioma [5]. Multipotent hemangioma-derived stem cells have been also suspected as a precursor cell for $\mathrm{IH}$ might $[5,6]$.

IH are characterized by a rapid endothelial cell proliferation phase (0-1 year), followed by an involution phase over the next several years of life, representing an unique ability to involute after proliferation, which often resolve without intervention or consequence [7] .

Although most of $\mathrm{IH}$ tend to regress spontaneously, between 10 and $15 \%$ are at risk for complications, especially during the proliferative phase $[7,8]$. IF can cause impairment of vital functions or permanent cosmetic disfigurement, while some subtypes rapidly develop complications, including pain, functional impairment, or bleeding [5, 7]. Ulceration is the most common complication of infantile hemangiomas and represents a therapeutic challenge [6]. In more than a half of the cases, the spontaneous regression leaves scars [9].

\section{Case Report}

A 4-month-old female patient born at term, from first, normal 


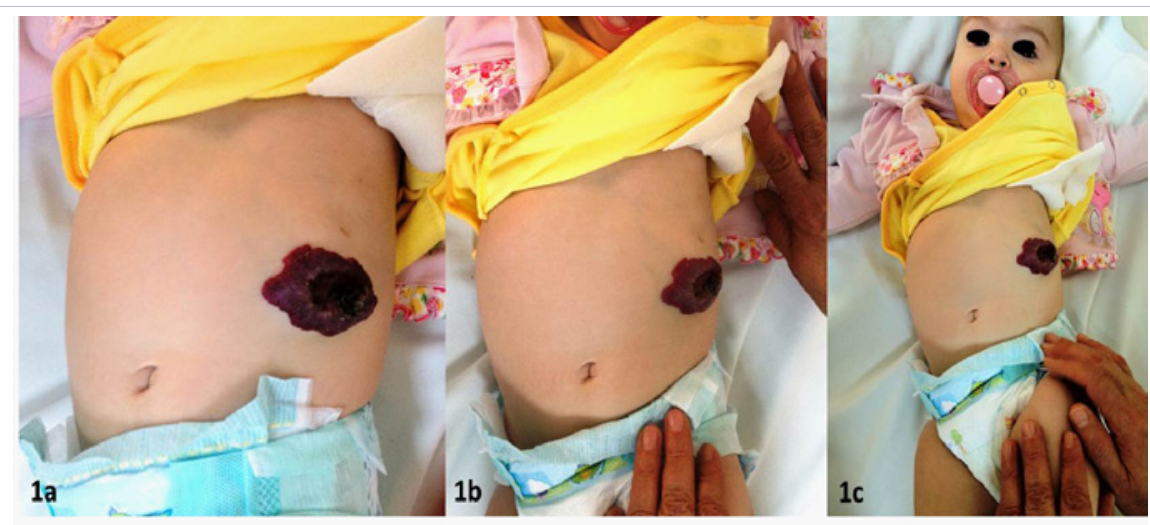

Figure 1: Large purple red, well-demarcated tumor with central ulceration, involving the left side of the abdomen.

proceeded pregnancy, without pathologies, presented with a giant ulcerated hemangioma, measuring $6 \mathrm{~cm}$ in diameter, localized in the left side of the abdomen- in the area of the left hypochondrium [Fig. 1a-c].

This lesion appeared during the first week of life and had been progressively enlarging, showing ulceration for the last 2 weeks, causing progressively discomfort and pain. No other similar skin lesions were observed. Ultrasound scan of the lesion was done to know the depth of the lesion. Additional laboratory investigations were within normal limits.

Because of the considerable dimensions, the great potential of rapid growth we've decided to initiate a topical treatment with Nebivolol gel (beta blocker), applied 5 times per day. The evolution was favorable without complications or adverse reactions.

\section{Discussion}

Clinical diagnosis of IF is usually easy, regarding the typical presentation of a mass or stains cutaneous red, of a subcutaneous mass or, generally, of a mixed form associating the two aspects [10]. Although that the evolution is mostly favorable with spontaneous regression in $80 \%$ of cases, a risk of sometimes progressive growth remains high [10]. Even if phase of stabilization and a phase of slow secondary regression could be seen within the first decade of life, the therapeutic challenge aim to prevent the risk of further complications, which could be often unpredictable [10].

Recent Wang $\mathrm{S}$ et al investigated the expression of $\mathrm{p} 16$ protein in $\mathrm{IH}$, detected by immunohistochemistry, observing that the expression level of p16 protein in the proliferating hemangioma was lower than that in the involuting hemangioma and that the expression level of p16 protein in the involuting hemangioma was lower than that in the normal tissue [11]. These results suggest that the $\mathrm{p} 16$ protein is present at a low level of expression in proliferating hemangioma and it cannot inhibit the hemangioma endothelial cell CDK4/CDK6 activity as a result, wich subsequently makes it unable to prevent the cell from entering the G1 phase to S phase transition [11]. Therefore, the cell proliferation becomes uncontrolled, leading to the excessive proliferation of the endothelial cells, accelerating hemangioma proliferation [11]. In contrast, the expression of p16 protein is increased within the period of IH regression, inhibiting the hemangioma endothelial cell CDK4/CDK6 activity, leading to cell cycle arrest [11]. The subsequent inhibition of the proliferation of the hemangioma endothelial cells promotes the involution of IH [11]. The significant difference between the results in the investigative lesions is a promising new entity in the differentiation between the IH capacity of proliferation and potential of rapid growth, which could result in further complication.

Although a new horizon has been estimated in the diagnosis, further investigations are still needed to confirm or reject this hypothesis. Meanwhile, the clinician must be aware of current therapeutic options, considering their indications, advanced reactions and therapeutic response. The treatment modalities of infantile hemangioma are: systemic steroids, intralesional corticosteroid injections, Interferon-alpha, vincristine, beta blockers, sclerosing agents, laser therapy, surgery or a combination of these therapies, considering the side effects of each one and also their ineffectiveness in some cases of IH $[5,7]$.

Former therapeutic options for complicated and ulcerated IH include systemic corticosteroid therapy at doses of 2 to 3 $\mathrm{mg} / \mathrm{kg} /$ day had $75 \%$. Unfortunately, long term steroid use is associated with side effects such as electrolyte abnormalities, cardiovascular and musculoskeletal disorders, adrenal suppression, immunosuppression and growth retardation $[5,6]$. Furthermore, regrowth of the hemangioma sometimes occurs after stopping the therapy with corticosteroids which makes them insufficient treatment option [6].

Management of IH has changed significantly after 2008 with use of propranolol. Although IH is not among the approved indications for $\beta$-blockers, propranolol is still recommended as the first line treatment for $\mathrm{IH}$, as the vasoconstrictors, regulating angiogenic pathways functions and inducing apoptosis of vascular endothelial cells activity are beneficial to the rapid regression of IH [12].

Recently, non-selective beta-blockers were introduced as an effective and good safety therapy and have revolutionized 
the treatment of IH $[13,14,15]$. Available studies have shown that oral and topical propranolol, topical timolol gel, nadolol and atenolol are highly effective in the treatment of IH. Propranolol it's the most reported beta-blocker used in the treatment of IH $[13,14,15,16]$. Various dosage schedules have been described for oral propranolol which ranges from 1 to $3 \mathrm{mg} / \mathrm{kg} /$ day divided into 3 doses. The mean response rate is approximately $98 \%$ [14]. A meta-analysis of 61 studies, involving 5,130 participants demonstrated that propranolol was more effective and safer than other therapies in treating IH [15]. Since 2012 a topical formulation of propanaol is available for small to medium sized IH's, were oral treatment is not inducated.

The therapeutic effects of propranolol on IH are vasoconstriction, declined expression of vascular endothelial growth factor and basic fibroblast growth factor genes and triggering of apoptosis [13]. The optimal duration of treatment has not yet been confirmed. Studies on the value of propranolol in IH treatment have shown good response after median treatment durations between 3 to 10.5 months $[13,14,15]$. The following adverse events were observed in patients using oral propranolol: mental depression manifested by insomnia, hypoglycemia, hypotension, bradycardia, congestive heart failure, bronchospasm and acrocyanosis. Rebound growth of lesion was noticed after discontinuation of therapy with propranolol [16]. However, compared with other treatment modalities propranolol is a relatively low risk medication $[15,16]$ which makes it the preferred treatment for problematic $\mathrm{IH}$, although that metaanalysis results shows complications rate of medium $31 \%$ of patients at a mean dosage of $2.12 \mathrm{mg} / \mathrm{kg} /$ day [17].

Recent study reports on the minimal dosage and duration of treatment to achieve good terapeutic response resulting in rapid involution of the lesion with minimal side effects using a stepwise escalation regimen, including administration of propranolol in divided doses of $1.5-2 \mathrm{mg} / \mathrm{kg} /$ day, with stepwise escalation, with treatment duration to an average age of 14.2 months [17].

Topical timolol maleate $0.5 \%$ gel, share the same mechanism of action as propranolol and is believed to have less systemic adverse effects. The early intervention will facilitate a faster resolution of the IH [16]. Surgery remains an appropriate treatment alternative in $\mathrm{IH}$ that are refractory to treatment or that need urgent treatment $[14,16]$. Even if there are many methods of treatment, evidence-based information about optimal medication and dosage, adverse effects, treatment modality, pretreatment and treatment strategies are still limited.

\section{References}

1. Sans V, de la Roque ED, Berge J, Grenier N, Boralevi F, MazereeuwHautier J, et al. Propranolol for severe infantile hemangiomas: follow-up report. Pediatrics. 2009;124(3):e423-431. doi: 10.1542/ peds.2008-3458. Epub 2009 Aug 10.

2. George A, Mani V, Noufal A. Update on the classification of hemangioma. J Oral Maxillofac Pathol. 2014;18(Suppl 1):S117-120. doi: 10.4103/0973-029X.141321.

3. Liang MG, Frieden IJ. Infantile and congenital hemangiomas. Semin Pediatr Surg. 2014;23(4):162-167. doi: 10.1053/j. sempedsurg.2014.06.017

4. Mulliken JB, Glowacki J. Hemangiomas and vascular malformations in infants and children: A classification based on endothelial characteristics. Plast Reconstr Surg. 1982;69:412-422.

5. Gey A, Ezzedine K, Diallo A, Prey S, Dreyfus I, Maza A, et al. Stay in NICU and infantile haemangioma development. Journal of the European Academy of Dermatology and Venereology. 2015;29:566-573. doi: 10.1111/jdv.12639.

6. Zheng JW, Zhang L, Zhou Q, Mai HM, Wang YA, Fan XD, et al. A practical guide to treatment of infantile hemangiomas of the head and neck. Int J Clin Exp Med. 2013;6(10):851-860.

7. Darrow DH, Greene AK, Mancini AJ, Nopper AJ. Diagnosis and Management of Infantile Hemangioma.Pediatrics. 2015;136(4):e1060104

8. Giachetti A, Sojo MM, García-Mónaco R. Infantile hemangiomas. Arch Argent Pediatr. 2013;111(6):537-545. doi: 10.1590/S032500752013000600014

9. Janmohamed SR, Madern GC, de Laat PC, Oranje AP. Educational paper: therapy of infantile haemangioma-history and current state (part II). Eur J Pediatr. 2015;174(2):259-266. doi: 10.1007/s00431014-2404-5.

10. Casanova D, Norat F, Bardot J, Magalon G. Cutaneous hemangioma: clinical aspects. Ann Chir Plast Esthet. 2006;51(4-5):287-292.

11. Wang S1, Zheng W2. Expression of p16 protein in infantile hemangioma. Oncol Lett. 2015;10(3):1589-1592.

12. Lin TT, He YJ. The advance of $\beta$-blockers in the treatment of infantile hemangiomas. Zhonghua Yan Ke Za Zhi. 2013;49(12):1138-1144.

13. Tiwari P, Pandey V, Gangopadhyay AN, Sharma SP, Gupta DK. Role of propranolol in ulcerated haemangioma of head and neck: a prospective comparative study. Oral Maxillofac Surg. 2016;20(1):7377. DOI: $10.1007 / \mathrm{s} 10006-015-0528 \mathrm{z}$

14. Pandey V, Tiwari P, Gangopadhyay AN, Gupta DK, Sharma SP, Kumar V. Propranolol for infantile haemangiomas: experience from a tertiary center. J Cutan Aesthet Surg. 2014;7(1):37-41. doi: 10.4103/09742077.129975

15. Liu X, Qu X, Zheng J, Zhang L. Effectiveness and Safety of Oral Propranolol versus Other Treatments for Infantile Hemangiomas: A Meta-Analysis. PLoS One. 2015;10(9):e0138100. doi: 10.1371/ journal.pone. 0138100

16. Raphael MF, Breur JM, Vlasveld FA, Elbert NJ, Liem YT, Kon M, et al. Expert Opin Drug Saf. 2016;15(2):199-214. doi: $10.1517 / 14740338.2016 .1130125$

17.Tan CE, Itinteang $\mathrm{T}$, Leadbitter $\mathrm{P}$, Marsh $\mathrm{R}$, Tan ST. Low-dose propranolol regimen for infantile haemangioma. J Paediatr Child Health. 2015;51(4):419-24. doi: 10.1111/jpc.12720. 